\title{
Modifying Graphene Substrates for Imaging Proteins in a Transmission Electron Microscope
}

\author{
Christopher J. Russo and Lori A. Passmore
}

Medical Research Council Laboratory of Molecular Biology, Cambridge United Kingdom.

Graphene is an ideal substrate for imaging single molecules in a transmission electron microscope [1, 2], but its hydrophobic nature precludes reliable protein deposition and has prevented its widespread adoption in electron cryo-microscopy (cryo-EM). We have developed a simple yet robust method of modifying graphene using low-energy plasmas so it is suitable for use with biological molecules. The method removes surface contaminants and reduces graphene hydrophobicity without degrading the substrate lattice. We use chemical vapor deposition to synthesize large quantities of graphene [3] and use it to make suspended graphene substrates. We then modify the suspended graphene to allow control of protein surface distribution in ice for cryo-EM. This enables the systematic screening of surfaces for protein adsorption. We show examples of controlled protein adsorption on graphene using a range of different protein complexes, including $70 \mathrm{~S}$ ribosomes, $80 \mathrm{~S}$ ribosomes, $20 \mathrm{~S}$ proteasomes and apoferritin. We demonstrate the use of graphene in high-resolution structure determination by cryo-EM and show that it consistently reduces the radiation-induced motion of individual protein complexes, thus improving the structural information content in each micrograph.

\section{References:}

[1] Geim, A. K. Science 324 (2009), p. 1530.

[2] Pantelic, R. S. et al. J Struct Biol 174 (2011), p. 234.

[3] Li, X. et al. Science 324 (2009), p. 1312.

[4] The authors thank S. Schotcher, A. Fowle, P. Heard and M. Kyte for fabrication of custom machines and electronics; I.S. Fernandez, A. Kelley, and V. Ramakrishnan for ribosomes; J. Grimmett, T. Darling, G. McMullan and S. Chen for technical assistance, R.A. Crowther, S. Scheres, and R. Henderson for helpful discussions. This work was supported by the European Research Council under the European Union's Seventh Framework Programme (FP7/2007-2013)/ERC grant agreement no 261151 to L.A.P., a MRC (UK) Centenary award to C.J.R. and MRC (UK) grant U105192715 (L.A.P.). 


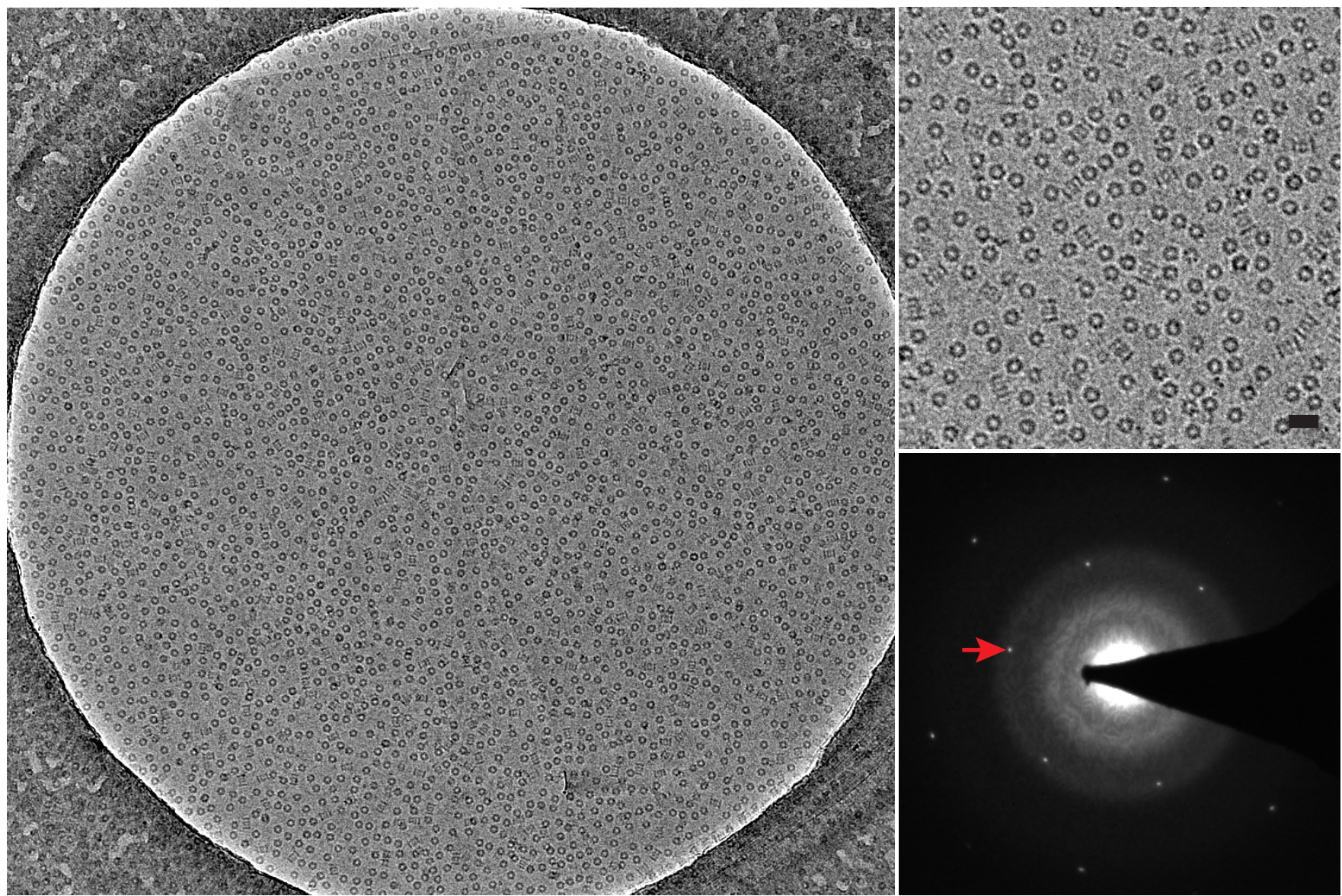

Figure 1. Human 20S proteasome protein particles adsorbed to monolayer graphene. Left panel shows graphene suspended across a micron sized hole that is uniformly covered with protein particles embedded in vitreous ice. Scale bar is $2500 \AA$. Upper right is a magnified view from left, scale bar is 250 $\AA$. Sample was imaged with $300 \mathrm{keV}$ electrons at $90 \mathrm{~K}$; fluence was $64 \mathrm{e} / \AA^{2}$. Lower right is the selected area diffractogram from the suspended region. Arrow points to the $0-110$ reflection of the graphene lattice, 2.1 $\AA$, which sets the scale for the diffraction pattern. The Debye-Scherrer rings of the amorphous ice in which the proteins are imbedded, at $3.7 \AA$ and $2.1 \AA$, are clearly visible. 